\title{
Astronomy
}

\section{Hollow meteoroid streams}

\section{from David W. Hughes}

IN December every year, meteors issue from the constellation of Gemini as Earth passes through the Geminid meteoroid stream. Radio meteoroids can be detected for about a week and the visual and photographic activity lasts slightly less long. Recent observations of the Geminid stream demonstrate that the conventional view of old meteor streams needs reconsidering. Instead of simply spreading as they age, the streams become hollow, literally forming pipes.

The Geminid meteor shower varies little from year to year, indicating that the stream is more than 500 years old. Recent observations with the Infrared Astronomical Satellite led to the detection of Asteroid 3200 , which has a very similar orbit to that of the Geminid stream. It is still a matter of speculation as to whether Asteroid 3200 is the degassed nucleus core of the stream's parent comet or whether the Geminid comet choked to death by building up too thick an insulating dust crust and now appears asteroid-like.

Streams are formed from dust particles which have been ejected from the parent cometary nucleus with a spread of velocities, and thus slowly gain on or fall behind the comet until a complete annulus of dust eventually surrounds the cometary orbit. The orbits of these dust particles are perturbed by planetary gravitational fields, radiation pressure, the PoyntingRobertson effect and interactions between their electric charges and the interplanetary magnetic field. The Geminid stream makes no close approaches to any of the major planets, so the effect of perturbation is relatively straightforward. Jupiter is the major perturber with Saturn, Earth and Venus providing 4.1, 2.6 and 2.3 per cent, respectively, of the jovian effect.

J. Jones started a perturbation analysis of the Geminid stream by considering only Jupiter (Mon. Not. R. astr. Soc. 217, 523; 1985). He replaced the stream by 71 meteoroids at random mean anomalies around the orbit, and then perturbed these particles by a point-mass Jupiter for a period of 5,000 years. He was expecting to find that the stream broadens with time with essentially a gaussian cross-section, but his results were "the causes of great astonishment". Instead of Gaussians, he found that the cross-sections are like those of a thick-walled, somewhat squashed, pipe which is flattened parallel to the plane of the ecliptic. The in-ecliptic width of the pipe increases with time (at $1 \mathrm{AU}$ from the Sun it is $0.006 \mathrm{AU}$ across after 1,000 years and $0.022 \mathrm{AU}$ across after
5,000 years, the other dimension reaching a minimum after 3,000 years but opening up again later). Adding the other planets to the calculation increases the crosssection of the pipe by a few per cent.

The Earth moves at about 0.017 Au per day around its orbit, so would take just over a day to cross a 5,000-year-old stream. If the Earth passes through a hollow stream, two brief periods of meteor activity would be observed, but as there is no reason why it should pass through the centre of the stream, the two bursts of activity would on average (assuming an elliptical cross-section) be separated by 64 per cent of the maximum possible time interval.

Has the predicted twin-peaked activity been observed for the Geminid stream? Radar data usually consist of many observations of faint meteors from a single station, whereas visual observations yield much lower fluxes but rely on many observers spaced over the globe. The addition of two, theoretical, separate gaussian activity curves convinced Jones that offsets of less than two days (corresponding to 16,000 years of perturbation) cannot be detected in radar fluxes from a single station. Combining results from nonidentical, spaced stations and from year to year (which include intrinsic variations of activity) was thought to be too risky.
As the visual meteor shower only lasts half as long as the radar one the twin-peak nature of the proposed flux should be more obvious. A detailed study of visual Geminid data between 1970 and 1979 (see G.H. Spalding, J. Br. astr. Ass. 92, 5; 1982) shows just such a bifurcation, a secondary maximum being clearly visible 0.8 days $(0.014 \mathrm{AU})$ after the main maximum. This observation provides evidence that the Geminid stream is about 3,000 years old.

Jones stresses two other points. In the early history of the development of a meteor stream, other processes could mask the influence of planetary gravitational perturbation, but as the spreading caused by this effect is directly proportional to the age of the stream it will eventually dominate. If the stream has its aphelion near the orbit of Jupiter the perturbations causing hollowing are even more pronounced. Four streams immediately spring to mind: the Taurids; the Delta Aquarids; the Piscids; and the Quadrantids. The first three have double maxima that have previously been designated as the northern and southern branches of the showers. Jones thinks the picture is much simpler; one branch is seen when the Earth enters the thick pipe of meteoroid dust, the other when it leaves. The Quadrantid shower is extremely narrow (a characteristic that indicates youthfulness) and is therefore too young to have become hollow.

David W. Hughes is Senior Lecturer in Astro. nomy and Physics at the University of Sheffield, Sheffield S3 TRH, UK.

\section{Ecological modelling}

\section{Invasions of natural communities}

\section{from James A. Drake and Mark Williamson}

INVADING species can affect natural communities and ecosystems in various ways. For example, the gypsy moth (Porthetria dispar) was introduced to North America in 1869; less than 100 years later it had defoliated more than 600,000 hectares of forest, and current predictions of damage to forest ecosystems in the northeastern United States are as high as 85 million hectares ${ }^{1}$. There are many other examples of the disruptive potential of non-native species.

The international working group on modelling species invasions of the scientific committee on problems of the environment (SCOPE-ICSU) met recently* to discuss and to synthesize approaches to modelling the invasion process. The group is part of a programme which is examining

*SCOPE working group on the modelling of the ecology of biological invasions, Fontana, North Carolina, 9 - 13 September 1985 . the ecological aspects of invasive species in natural and semi-natural ecosystems. It is to be hoped that accurate modelling of the invasion processes will lead to the development of cost-effective control measures; an understanding of the mechanisms governing species invasions also is essential if genetically engineered species are soon to be released into the environment ${ }^{2}$.

Species invasion involves at least three distinct stages: arrival, establishment and dispersal. Elucidation of arrival and establishment mechanisms is the necessary first step in the modelling process; both deterministic and stochastic models are likely to be valuable (Stuart Pimm, University of Tennessee). Species movement throughout the biosphere is strongly influenced by patterns of human travel, and many more species are now candidates for longdistance dispersal than in the past. Pat- 